\title{
28 Research Square \\ Efficacy of Acupuncture and Related Techniques on Delirium: Protocol of a Systematic Review and Meta-analysis
}

\section{Sulan Tan}

LZMC: Southwest Medical University https://orcid.org/0000-0001-5509-8883

\section{Yu Zhang}

Southwest Medical University

\section{Yiping Bai}

Southwest Medical University

Shuting Yang

Sun Yat-Sen Memorial Hospital

\section{Liming Luan}

Vanderbilt University Medical Center

Jun Zhou (D634599002@qq.com)

Department of Anesthesiology, Affiliated Hospital of Southwest Medical University

\section{Protocol}

Keywords: Delirium, Acupuncture, Acupoint, Transcutaneous electrical acupoint stimulation

Posted Date: January 15th, 2021

DOI: https://doi.org/10.21203/rs.3.rs-144659/v1

License: (c) (1) This work is licensed under a Creative Commons Attribution 4.0 International License. Read Full License 


\section{Abstract}

Background: Delirium, contributing to subsequent increased morbidity and mortality, is common in hospitalized patients, especially in patients undergoing major surgery. Published systematic reviews suggest that certain medications for delirium management may decrease pain scores and analgesic requirements, at the expense of troublesome side effects. People are urgently seeking non-medication strategies for delirium. Acupuncture and related techniques (ART) are increasingly used to provide medical care and have potential to prevent delirium, however, the efficacy of ART on delirium remains controversial.

Methods: In accordance with the PRISMA-P guidelines, we will search PubMed, Embase, the Cochrane Library (Cochrane Central Register of Controlled Trials (CENTRAL)), Web of Science, Medline and the Cochrane Library for clinical trials on ART use in people, without language restrictions. The incidence of delirium will be defined our primary outcome. Additional outcomes will be the incidence of adverse events, pain score, consumption of analgesics, number of cumulative delirious days, hemodynamics, length of hospital stay. Two researchers independently complete further review work and data abstraction based on pre-specified inclusion and exclusion criteria. Any discrepancy will be solved by negotiation or a third investigators. Cochrane Risk of Bias tool will be used to assess the methodological quality of all the studies that are finally included. Statistical heterogeneity was assessed by forest plots, confidence intervals $(\mathrm{Cl})$ and $\mathrm{I}^{2}$ statistic. If feasible, a meta-analysis of included results will be performed.

Conclusions: This protocol will be favorable to bridge the gap between non-medication strategies and clinical diseases, and potential to help future guideline development in management of preventing delirium.

Systematic review registration: The protocol was prospectively registered on the homepage of the International Prospective Register of Systematic Reviews (PROSPERO):

https://www.crd.york.ac.uk/PROSPERO.

\section{Background}

The frequency of delirium in hospitalized patients ranges from $15-50 \%$, and it is even up to $83 \%$ in the intensive care unit (ICU) [1]. Delirium is a big challenge to patients in terms of prolonged hospital stay, financial burden, mortality and morbidity [2], as well as medical care team's clinical assessments, increased workload [3]. Several medications have been clinically introduced to prevent delirium [4-6]. Considering that each medicine has its disadvantages especially in terms of efficacy, this approach seems to be controversial [7]. Recently, Bolan JW suggested that medications were not generally recommended as some of them could exacerbate delirium [8]. Consequently, it may be time for people focus more on alternative non-pharmacological interventions for delirium.

Acupuncture, as an integral part of the ancient Chinese medical system, has been used to treat diseases over 2,500 year [9]; currently, acupuncture-points stimulation, as an emerging field in modern medical 
care, has potential to restore physiological homeostasis [10]. Acupuncture and related techniques (ART), including invasive methods (skin penetration with an acupuncture needle) and noninvasive methods (acupressure, transcutaneous electrical stimulation, moxibustion, and application of various stimulating patches and pellets) [9], are increasingly used in modern medicine for pain relief, antidepressant effect, and ejaculation-delaying [11-13]. Moreover, transcutaneous electrical acupoint stimulation has been approved by the the American Pain Society, National Institutes of Health $(\mathrm{NIH})$, and the World Health Organization (WHO) [14, 15]. Recent advances in clinical experimental research of acupuncture provided new information on the cognitive disorders to inhibit neuroinflammation, and this approach has been reported to be effective in populations of children and adults $[16,17]$.

However, the safety and efficacy of acupuncture on delirium is much of controversial, because its successful implementation is largely constrained by the experience of the practitioners and the scarcity of mechanistic studies. Regrettably, to the best of our knowledge, there is currently no systematic review or meta-analysis of this issue. Thus, the purpose of this systematic review protocol is to evaluate the efficacy of ART in the management of delirium, and to provide a possible ideal strategy to reduce the incidence of delirium.

\section{Methods}

\section{Aims}

The primary objective of this investigation is to evaluate the efficacy of acupuncture on delirium compared with placebo or any other interventions. Other outcomes are to assess its effect in different ways of acupoint stimulation, the incidence of adverse events, pain score, consumption of analgesics, number of cumulative delirious days, hemodynamics, as well as the length of hospital stay.

\section{Design}

The implementation of our systematic review will rigorously comply with the methodological recommendations of the Cochrane Handbook for Systematic Reviews and Meta analyses and the items of Preferred Reporting Items for Systematic Review and Meta-Analysis Protocols (PRISMA-P) [18], and the checklist is available in Additional file 1. The protocol was prospectively registered on the homepage of the International Prospective Register of Systematic Reviews (PROSPERO).

\section{Eligible criteria}

Eligible criteria are determined based on PICOS (Population, Intervention, Comparator, Outcomes and Study Design) guidance, with detailed information presented in Table 1.

\section{Information sources and search strategy}

In order to obtain the most comprehensive literature, we will search PubMed, Embase, the Cochrane Library (Cochrane Central Register of Controlled Trials (CENTRAL)), Web of Science, Medline and the Cochrane Library, without language restrictions. We will split the search content into three core term 
categories (intervention (acupuncture, acupuncture needle, acupressure, transcutaneous electrical stimulation, moxibustion, acupressure, stimulating patches and pellets), outcome (delirium, postoperative delirium, POD), RCTs) to generate medical subject terms (MeSH) and free-text terms to retrieve literature. The search strategy will be verified by an experienced information specialist, which will be adapted for the aforementioned databases. Next, we will download all the search results and upload them to the literature management software, and then delete duplicate data automatically or manually.

\section{Study selection}

Two independent researchers will conduct the screening work in two stages in lines with the preestablished inclusion and exclusion criteria. In the first stage, the titles and/or abstracts will be scanned to remove items that do not meet the criteria, and then, qualified studies will go through full text review stages. Any disagreements arising at any step will be resolved instantly after negotiation or a third party.

\section{Data abstraction}

We will first establish a standardized data extraction accepted by the review team, in the form of excel. Data will be extracted by two members separately. Instead of directly compiling the extracted data, the data will be placed in a separate excel first. Two members will participate in reviewing all selections to reach an agreement. Disagreements will be settled by negotiation, and if it fails, the third member will be required to mediate. The study authors will be contacted for data clarification or insufficient data. Finally, all valid data will be aggregated into an individual master sheet. When the results are presented in the form of graphs, we will use an application (WebPlotDigitizer) [19] to extract the data.

We will extract the following main characteristics of all included studies:

- Study characteristics: First author, publication year, country, total sample size, the number of patients analyzed in each group;

- Patients characteristics: age, sex, prior chronic use of sedatives and dependence, whether the operation (type of surgery and anesthesia), type of population (geriatric or not);

- Intervention and comparators: the acupoints, type, timing, the duration of treatment;

- Outcomes: delirium (assessment tools, timing of evaluation), others (the incidence of adverse events, pain score, consumption of analgesics, number of cumulative delirious days, hemodynamics, the length of hospital stay)

\section{Risk of bias assessment}

Two researchers will be appointed to use Review Manager's (version 5.3) built-in Cochrane risk of bias assessment tool to separately evaluate the methodological quality of the final inclusion studies, which involved seven different domains:

- random sequence generation (selection bias)

- allocation concealment (selection bias) 
- blinding of participants and personnel (performance bias)

- blinding of outcomes assessment (detection bias)

- incomplete outcome data (attrition bias)

- selective reporting (reporting bias)

- other bias.

Each trial was classified as high risk or unclear or low risk after rigorous analysis. The result will finally be displayed graphically.

\section{Data synthesis}

Stata 12.0 (StataCorp LLC, College Station, USA) will be executed to pool and analyze the extracted data. Our primary outcome is the incidence of delirium, which can be determined in advance as dichotomous data, so when processing this data, the relative risk (RR) (or odds ratio (OR) if deemed appropriate) and a 95\% confidence interval $(\mathrm{Cl})$ will represent the measure of effect size. But for other outcomes, effect size will be presented by specific data types, for continuous data, we will take the mean difference (MD) (or standardized mean difference (SMD) in case of different evaluation scale) with $95 \%$ confidence intervals [20]. Based on the random effects model, we use the I-squared statistics to quantify the significant heterogeneity. The heterogeneity will be identified considerable if $\mathrm{I}^{2}$ is more than $50 \%$ [21], and the effect model remains unchanged. Otherwise, we will consider replacing it with a fixed effects model. In addition, we will conduct sensitivity analysis and subgroup analysis to confirm the robustness of the results and attempt to find the sources of heterogeneity. Literature that can explain the significantly heterogeneity will be eliminated and narrated separately. Subgroup analysis will be carried out considering participants (gender, region, surgery), types of anesthesia (general anesthesia, spinal anesthesia or others), interventions (intervention methods (non-invasive or invasive), intervention timing), outcome (delirium assessment tool, assessment time). Meta-regression with multiple covariates will be performed, if possible. Moreover, we will conduct the trial sequential analysis (TSA) to minimize false positive results due to sparse sample size and random errors [22]. Publication bias will be assessed by funnel plots and Egger's test ( $p$-value $>0.5$ means no significant asymmetry), if sufficient data are available. The strength of the evidence will be assessed in accordance with the Grading of Recommendations, Assessment, Development, and Evaluation (GRADE) guidelines [23], and the level of judgment is targeted is on the summary of the evidence.

\section{Discussion}

Pharmacological treatment of delirium has always been faced with distressing problems in clinical practice, such as the uncertainty of the effect of multiple reagents combinations and the considerable health care costs [7]. Some scholars have proposed that certain drugs may even exacerbate the patients' condition [8]. Several studies had reported that acupuncture or electroacupuncture could inhibit inflammation and protect organs from infectious and inflammatory disorders [24, 25], and the inflammatory theory is involved in occurrence of delirium [26]. In addition, proper acupuncture could 
provide good analgesia and reduce the consumption of analgesics. It has been proven that pain is one of the high-risk factors leading to delirium [27, 28]. Moreover, a correlation between the corresponding activation of specific areas of the brain and the acupoint stimulation was confirmed by Cho et al. using functional MRI [29]. The above description shows that acupuncture has great potential for preventing delirium. But the underling mechanism of acupuncture against delirium remains controversial. Certain effective and feasible non-pharmaceutical interventions to prevent delirium urgently need to be confirmed. Recently, Deng LX et al. did a network meta-analysis to evaluate a variety of nonpharmacological interventions to prevent delirium [30]. Unfortunately, acupuncture and related techniques were not included in this research. Our review will focus on the effectiveness of acupuncture on delirium, and provide practical and research significance for the future. If the expected positive results can be achieved, and then implemented in the clinical environment, not only can effectively decrease the incidence of delirium, but also reduce the economic burden.

\section{Abbreviations}

ART: Acupuncture and related techniques; PRISMA-P: Preferred Reporting Items for Systematic Review and Meta-Analysis Protocols; Cl: Confidence interval; ICU: intensive care unit; $\mathrm{NIH}$ : National Institutes of Health; WHO: World Health Organization; PICOS: Population, Intervention, Comparator, Outcomes and Study Design; POD: postoperative delirium; RCT: Randomized control trials; RR: Relative risk; OR: Odds ratio; MD: Mean difference; SMD: Standardized mean differences; TSA: Trial sequential analysis

\section{Declarations}

\section{Acknowledgements}

Not applicable.

\section{Authors' contributions}

ST, YZ, and FL were involved in the design of the protocol and the subsequent draft. YB and JZ participated in the review and revision of the draft, and provided the final version of the manuscript, which has been approved by all authors. JZ is the guarantor.

\section{Funding}

This work was supported by National Natural Science Foundation of China (No. 81873930).

\section{Availability of data and materials}

All data and materials generated or analyzed during this research are available on request.

\section{Ethics approval and consent to participate}


Not applicable.

\section{Consent for publication}

Not applicable.

\section{Competing interests}

None.

\section{Conflicts of interest}

none.

\section{References}

1. Ely EW, Bernard GR, Speroff T, Gautam S, Dittus R, May L, et al. Delirium in mechanically ventilated patients: Validity and reliability of the Confusion Assessment Method for the intensive care unit (CAM-ICU). JAMA. 2001 Dec 5;286(21):2703-10.

2. Arinzon Z, Peisakh A, Schrire S, Berner YN. Delirium in long-term care setting: Indicator to severe morbidity. Arch Gerontol Geriatr. May-Jun 2011;52(3):270-5.

3. Lawlor PG, Bush SH. Delirium in patients with cancer: Assessment, impact, mechanisms and management. Nat Rev Clin Oncol. 2015 Feb;12(2):77-92.

4. Keles S, Kocaturk O. Comparison of oral dexmedetomidine and midazolam for premedication and emergence delirium in children after dental procedures under general anesthesia: A retrospective study. Drug Des Devel Ther. 2018 Mar 28;12:647-653.

5. Duan X, Coburn M, Rossaint R, Sanders RD, Waesberghe J V., Kowark A. Efficacy of perioperative dexmedetomidine on postoperative delirium: systematic review and meta-analysis with trial sequential analysis of randomised controlled trials. Br J Anaesth. 2018 Aug;121(2):384-397.

6. Agar MR, Lawlor PG, Quinn S, Draper B, Caplan GA, Rowett D, et al. Efficacy of oral risperidone, haloperidol, or placebo for symptoms of delirium among patients in palliative care: A randomized clinical trial. JAMA Intern Med. 2017 Jan 1;177(1):34-42.

7. Deiner S, Luo X, Lin HM, Sessler DI, Saager L, Sieber FE, et al. Intraoperative infusion of dexmedetomidine for prevention of postoperative delirium and cognitive dysfunction in elderly patients undergoing major elective noncardiac surgery : A randomized clinical trial. JAMA Surg. 2017 Aug 16;152(8):e171505.

8. Boland, J. W., Lawlor, P. G., \& Bush, S. H. (2019). Delirium: non-pharmacological and pharmacological management. BMJ Support Palliat Care. 2019 Dec;9(4):482-484.

9. Chernyak G V., Sessler DI. Perioperative acupuncture and related techniques. Anesthesiology. 2005 May;102(5):1031-49; quiz 1077-8. 
10. Ulloa L, Quiroz-Gonzalez S, Torres-Rosas R. Nerve Stimulation: Immunomodulation and Control of Inflammation. Trends Mol Med. 2017 Dec;23(12):1103-1120.

11. Paley CA, Johnson MI, Tashani OA, Bagnall AM. Acupuncture for cancer pain in adults. Cochrane Database Syst Rev. 2015 Oct 15;2015(10):CD007753.

12. Smith CA, Armour M, Lee MS, Wang LQ, Hay PJ. Acupuncture for depression. Cochrane Database Syst Rev. 2018 Mar 4;3(3):CD004046.

13. Sunay $D$, Sunay $M$, Aydoğmuş $Y$, Bağbanci $S$, Arslan $H$, Karabulut $A$, et al. Acupuncture versus paroxetine for the treatment of premature ejaculation: A randomized, placebo-controlled clinical trial. Eur Urol. 2011 May;59(5):765-71.

14. Chou R, Qaseem A, Snow V, Casey D, Cross TJ, Shekelle P, et al. Diagnosis and treatment of low back pain: A joint clinical practice guideline from the American College of Physicians and the American Pain Society. Ann Intern Med. 2007 Oct 2;147(7):478-91.

15. Kong J, Spaeth R, Cook A, Kirsch I, Claggett B, Vangel M, et al. Are All Placebo Effects Equal? Placebo Pills, Sham Acupuncture, Cue Conditioning and Their Association. PLoS One. 2013 Jul 31;8(7):e67485.

16. Ochi JW, Richardson AC. Intraoperative pediatric acupuncture is widely accepted by parents. Int J Pediatr Otorhinolaryngol. 2018 Jul;110:12-15.

17. Ho YS, Zhao FY, Yeung WF, Wong GTC, Zhang HQ, Chang RCC. Application of Acupuncture to Attenuate Immune Responses and Oxidative Stress in Postoperative Cognitive Dysfunction: What Do We Know So Far? Oxid Med Cell Longev. 2020 Feb 13;2020:9641904.

18. Moher D, Shamseer L, Clarke M, Ghersi D, Liberati A, Petticrew M, et al. Preferred reporting items for systematic review and meta-analysis protocols (PRISMA-P) 2015 statement. Syst Rev. 2015 Jan 1;4(1):1.

19. Rohatgi A. WebPlotDigitizer. Available from: https://automeris.io/ WebPlotDigitizer/images/wpd.png. [Accessed 15 Aug 2017].

20. Hozo SP, Djulbegovic B, Hozo I. Estimating the mean and variance from the median, range, and the size of a sample. BMC Medical Research Methodology. BMC Med Res Methodol. 2005 Apr 20;5:13.

21. Higgins JPT, Thompson SG, Deeks JJ, Altman DG. Measuring inconsistency in meta-analyses. BMJ. 2003 Sep 6;327(7414):557-60.

22. Wetterslev J, Thorlund K, Brok J, Gluud C. Trial sequential analysis may establish when firm evidence is reached in cumulative meta-analysis. J Clin Epidemiol. 2008 Jan;61(1):64-75.

23. Balshem H, Helfand M, Schünemann HJ, Oxman AD, Kunz R, Brozek J, et al. GRADE guidelines: 3 . Rating the quality of evidence. J Clin Epidemiol. 2011 Apr;64(4):401-6.

24. Grech D, Li Z, Morcillo P, Kalyoussef E, Kim DD, Bekker A, et al. Intraoperative Low-frequency Electroacupuncture under General Anesthesia Improves Postoperative Recovery in a Randomized Trial. J Acupunct Meridian Stud. 2016 Oct;9(5):234-241. 
25. Torres-Rosas R, Yehia G, Peña G, Mishra P, Del Rocio Thompson-Bonilla M, Moreno-Eutimio MA, et al. Dopamine mediates vagal modulation of the immune system by electroacupuncture. Nat Med. 2014 Mar;20(3):291-5.

26. Van Munster BC, Aronica E, Zwinderman AH, Eikelenboom P, Cunningham C, De Rooij SEJA. Neuroinflammation in delirium: A postmortem case-control study. Rejuvenation Res. 2011 Dec;14(6):615-22.

27. Bilotta F, Lauretta MP, Borozdina A, Mizikov VM, Rosa G. Postoperative delirium: Risk factors, diagnosis and perioperative care. Minerva Anestesiol. 2013 Sep;79(9):1066-76.

28. Heo DY, Hwang BM. Intravenous patient-controlled analgesia has a positive effect on the prognosis of delirium in patients undergoing orthopedic surgery. Korean J Pain. 2014 Jul;27(3):271-7.

29. Cho ZH, Oleson TD, Alimi D, Niemtzow RC. Acupuncture: The search for biologic evidence with functional magnetic resonance imaging and positron emission tomography techniques. J Altern Complement Med. 2002 Aug;8(4):399-401.

30. Deng LX, Cao L, Zhang LN, Peng XB, Zhang L. Non-pharmacological interventions to reduce the incidence and duration of delirium in critically ill patients: A systematic review and network metaanalysis. J Crit Care. 2020 Dec;60:241-248.

\section{Supplementary Files}

This is a list of supplementary files associated with this preprint. Click to download.

- Additionalfile1.docx 